\title{
HR specialists' perceptions of the desirability of psychopathic traits in job candidates
}

\author{
Alexandru TUDOSOIU \\ The Bucharest University of Economic Studies, Bucharest, Romania \\ Valentina Mihaela GHINEA \\ The Bucharest University of Economic Studies, Bucharest, Romania \\ valentina_ghinea@yahoo.com \\ Ramona Elena CANTARAGIU \\ The Bucharest University of Economic Studies, Bucharest, Romania \\ ramona_cantaragiu@yahoo.com
}

PICBE | 728

\begin{abstract}
The study of psychopathy in organizational settings is still in its incipient stage, but scholars have already proven that the prevalence of psychopathic features in the corporate environment, especially in senior positions, is higher than in the general population (3\% compared with 1\% in general population) and that employees with psychopathic tendencies have a negative impact on the sustainability of organizations. It has been argued that human resources specialists have to become astute at identifying employees with psychopathic features in order to be able to manage their careers in a way that would not affect the organization's wellbeing. However, research has also proved that there are several psychopathic traits which make job candidates likelier to obtain a job, due to the positive impression that they create during the job interview. The study tested the desirability of psychopathic traits in job candidates from the perspective of human resources specialists and, based on a survey of a sample of 68 Romanian human resources specialists, found that candidates displaying an ability to remain calm under pressure, self-confidence and persuasiveness are more likely to be hired. Moreover, the study concluded that Romanian human resources professionals are somewhat aware of the threat posed by psychopathic employees and that most of the employers they represent use selection methods able to filter out potential psychopaths. The findings open up new avenues of research on the ways in which selection strategies can be employed in order to enable human resources specialists to effectively identify candidates with psychopathic tendencies.
\end{abstract}

Keywords: psychopathy, organization, selection process, human resources.

\section{Introduction}

In popular culture, psychopathy is usually associated with criminals who make the subject of the evening news and who are confined in prisons or in mental asylums. In spite of blockbuster movies such as American Psycho (2000), Silence of the Lambs (1991) or Hannibal (2013-2015) which portray psychopaths in a realistic manner (i.e., charming, intelligent, manipulative individuals who lack emotions like fear or anxiety and have no sense of remorse), it is difficult for the average individual to come to grips with the reality that between $0.5 \%$ and $2 \%$ of the general population is formed of psychopaths (Millon et al., 1998). In fact, the world is filled with functional psychopaths, i.e. individuals who display some of the characteristic features of psychopathology, but who are well integrated into society and, most importantly, very successful in their professions (Boddy, 2011). In addition, scandals such as Enron and the Wall Street economic crash have brought the topic 
of psychopathy in the business world to the attention of the media, where a myriad of newspaper articles, blogs and magazines blur the lines between scientific facts and popular culture (Deresiewicz, 2012) by offering advice on how to identify psychopaths and how to deal with them in the workplace without consulting the few available studies on corporate psychopaths (Smith \& Lilienfeld, 2012).

The earliest mentioning of psychopaths dates back to the $3^{\text {rd }}$ century B.C. and belongs to Theophrastus, a Greek philosopher who described several moral types including that of the "unscrupulous man". However, the term received its first medical definition in 1801, when Phillipe Pinel, a French psychiatrist introduced a new type of mental disorder ("manie sans délire" or mania without delusions) which inspired American psychiatrists Benjamin Rush and J.C. Pritchard to introduce two new terms: "alienation of the mind" and "moral insanity/moral imbecility" to describe individuals uncapable of normal emotional responses (Millon et al., 1998). However, it was the German psychiatrist J.L. Koch (1892) the one who first used the term "psychopathic" to describe a wide range of disorders including neuroses and mental retardation. The diverse and sometimes contradictory definitions of psychopathy fueled controversies over definitions and treatments, until 1941 when Hervey Cleckley introduced a list of 16 criteria for diagnosing psychopathy (e.g., egocentrism, lack of emotions or regret, charisma etc.) which inspired the definition of psychopathy included in the second edition of the American Psychiatrist Association's manual. Further refinements to the definition continued to be added, but even today there is little consensus over the diagnosis for psychopathy (Skeem et al., 2011).

The scholarly disagreements on the quantification of emotions or the lack of emotional responses has made it impossible for psychopathy to be included in DSM-V, the latest manual for the diagnosis of mental disorders. However, there are several clinician rating scales that can be used to study psychopathy. The Psychopathy Checklist-Revised (PCL-R) is a revised version of the checklist introduced by Hare $(2003,1980)$ which consists of 40 personality characteristics related to psychopathy. Further research led scholars to the development of various other scales, such as the Psychopathic Personality Inventory created using eight factors (Lilienfeld \& Andrews, 1996) and later reorganized into two main categories: fearless dominance (including social potency, stress immunity, fearlessness) and self-centered impulsivity (including carefree nonplanfulness, impulsive nonconformity, Machiavellian egocentricity, blame externalization) to which the authors also added a separate scale for cold-heartedness (Lilienfeld \& Widows, 2005). Later, Patrick et al. (2009) aimed to create an all-encompassing conceptualization of psychopathy, i.e. the Triarchic Model which was based on three main observable characteristics: boldness, disinhibition and meanness. Although it did not resolve the disputes regarding the definition of psychopathy, it did manage to draw attention to the fact that psychopathy is a constellation of traits, rather than a specific, yes/no diagnosis as in the case of PCL-R and PPI (Skeem et al., 2011).

\section{Literature review: Psychopaths within organization}

Latest research has shown that certain careers are more likely to be pursued by psychopaths (e.g., CEO, lawyer, sales person, surgeon, journalist etc.) due to the fact that they reward decision made on logic and are performed in organizations with hierarchical structures which allow a mixture of power and control over people (Dutton, 2012). Moreover, the prevalence of psychopathic features in the corporate environment, especially in senior 
positions, is higher than in the general population (3\% compared with $1 \%$ in general population) (Babiak, et al., 2010). The current corporate cultures which place increasing emphasis on making hard decisions, ability to resist stress and high competitiveness represent an environment where psychopaths can use their abilities to the fullest and thrive. However, although psychopaths are successful at their jobs, on the long term, there is the possibility for the organization to forget its moral compass, especially when these employees reach top positions, threatening not only the sustainability of the organization, but also that of the community which it is supposed to serve (Boddy et al., 2010a, 2010b). Thus, it is important for organizations to recognize psychopaths in their early career stages, but this is difficult, especially if their selection process does not include background checks or psychological tests. In fact, due to their charming and manipulative personalities, psychopaths can easily pass job interviews by wooing recruiters (Babiak \& Hare, 2006).

Nonetheless, once they are within the organization, there are several signs that can help human resources specialist to identify psychopathic employees. According to Faggioni and White (2009) and Babiak and Hare (2006), psychopathic employees adopt a behavior characterized by: a) pathological lying in the pursuit of their own agenda (stealing, sabotaging, gossiping etc.); b) unrealistic expectations followed by public humiliation of others and no recognition of fault; c) different techniques for spreading conflict and mistrust within the organization (social isolation, harassment, bullying etc.). Based on the available studies, it is evident that psychopaths have a negative impact on a variety of aspects of the organizational life. For example, due to their manipulative and egotistic behavior, they contribute to organizational conflicts, stress and bullying, which, in turn, lower staff morale, increase absenteeism and staff turnover (Boddy, 2011). These, combined with a disregard for corporate ethics and an unfair treatment of the lower ranks, threaten not only the performance of the organization, but also its survival (Boddy et al., 2010a). However, few studies have also shown that certain psychopathic features can have a beneficial effect on organizational life. For example, boldness is tied with heroic and altruistic behaviors, and, although heroism is also positively correlated with antisocial behaviors (Smith et al., 2013), this proves that certain psychopathic traits, in moderation, can have positive effects on organizational life and performance (Board \& Fritzon, 2005). Thus, it is important for human resources specialists to be able to properly measure the psychopathic traits of the employees in order to be able to manage those who display those traits that can be beneficial to the organization, and to avoid giving leadership positions to the others.

Considering that it is difficult to manage psychopaths once they ascend the corporate ladder, it is preferable for human resources specialists to identify these individuals during the selection process, which means that organizations need to be aware of the threat posed by psychopaths and have sound selection strategies to avoid hiring employees that do not fit the corporate values. The effectiveness of the hiring process is directly connected to company performance (Ballantine, 2009), and selecting an unfit person for the job eventually affects all the layers of the organization, starting from the bottom (team dynamics, motivation and performance) and climbing up to the company's performance on the market (turnover cost, recruitment and selection costs, loss of customers) (Bratton \& Gold, 2012). For the selection process, most companies use interviews sometimes combined with assessment centers, depending on the job and the available resources, but this approach is minimally effective at filtering functional psychopaths (Faggioni \& White, 2009). According the latest research, it is 
recommended to use as a selection tool, at least for managerial positions, a personality test designed to measure psychopathic traits such as the PPI-R which, although costly, is easily interpretable by non-clinicians. In addition, trial periods allow human resources specialists the opportunity to observe the behavior of the employees and to gather feedback from colleagues and superiors, thus allowing the identification of any psychopathic tendencies.

\section{Methodology}

The purpose of this quantitative study is to explore the behavior of HR professionals when faced with a job candidate that displays psychopathic traits. The study aims to test three main hypotheses:

H1: Certain psychopathic traits represent an advantage for the candidate during the job interview.

H2: The field of activity of the company influences the preference for certain psychopathic traits in candidates.

H3: HR professionals are able to handle the threat posed by psychopaths to their organizations.

The survey contained 25 questions: five socio-demographic questions (age, gender, HR experience, seniority, field), fifteen questions regarding the psychopathic traits that are valued by interviewers, and five questions regarding the methods used by interviewers to filter out psychopaths. Data was gathered with the help of the Typeform platform from Romanian HR practitioners or people who were responsible for recruitment and selection in small companies (e.g., managers) identified through the help of a Facebook group, i.e. HR HUB, which has more than 2,500 members or through the snowballing technique (i.e., recommendations). The participants had to be employed in companies working in field of professional services (i.e., consultancy, accounting, financial planning, investments etc.) or in the creative industries (i.e., advertising, marketing, publishing, design etc.). According to research, professional services might be more suitable for psychopathic employees in comparison to creative industries where organizations have flatter structures and less power is given to the individual (Dutton, 2012). The respondents were informed about the aim of the study, but the terms used to describe the psychopathic traits were taken from the Psychopathic Personality Inventory (Lilienfeld \& Widows 2005) and modified in order to ensure objectivity and understandability:

Table 1. The rephrasing of the psychopathic terms

\begin{tabular}{|c|c|}
\hline Rephrased terms & Original traits (PPI) \\
\hline Cool under pressure & Stress immunity \\
\hline Confident & Machiavellian egocentricity \\
\hline Persuasive & Social potency \\
\hline Charismatic & Social potency \\
\hline Adventurous & Fearlessness \\
\hline Relaxed & Carefree nonplanfulness \\
\hline Egocentricity & Machiavellian egocentricity \\
\hline
\end{tabular}

Source: Author's interpretation on the traits described in the Psychopathic Personality Inventory. 
The final sample was formed of 68 participants, out of which 52 (76\%) were working in the professional service field, and the other 16 in creative industries. The majority of the respondents were women (87\%) with ages between 25 and $35(71 \%)$ and with a seniority of less than 3 years (72\%). The level of expertise varied widely from 17 participants with less than 3 years of experience, followed by 14 people with 3 to 5 years of experience, 13 people with 5 to 10 years of experience, and ending with 11 seniors with more than 10-year experience. There were also 7 participants who did not work in HR and only carried job interviews.

\section{Results and discussions}

\section{Psychopathic characteristics that advantage candidates during interviews}

Psychopaths are very skilled at making a good first impression (Babiak \& Hare, 2006), and, if the interviewer is impressed by the candidate at the beginning of the interview, he might not be able to spot the candidate's shortcomings later on in the interview. Thus, we asked participants to assess the importance of first impressions. The average score was 3.87, with over half of the people rating first impressions as important (51\% voted for "important" - 4) and $26 \%$ voted for "mildly important" - 3.

Next, respondents were asked to rank the top three desirable characteristics of job candidates from 3 batches each with 5 characteristics (out of which 2 related to psychopathy) and the results are displayed in Table 2.

Table 2. The visibility of the psychopathic traits

\begin{tabular}{|c|c|c|c|c|c|c|c|c|c|c|}
\hline & \multicolumn{7}{|c|}{ Traits and percentages obtained } \\
\hline $\begin{array}{c}\text { Batch } \\
1\end{array}$ & Honest & $88 \%$ & Efficient & $79 \%$ & Confident & $66 \%$ & Intuitive & $38 \%$ & Charismatic & $26 \%$ \\
\hline $\begin{array}{c}\text { Batch } \\
2\end{array}$ & Flexible & $91 \%$ & $\begin{array}{c}\text { Cool under } \\
\text { pressure }\end{array}$ & $87 \%$ & Meticulous & $53 \%$ & Competitive & $50 \%$ & Adventurous & $16 \%$ \\
\hline $\begin{array}{c}\text { Batch } \\
3\end{array}$ & Cooperative & $88 \%$ & Independent & $81 \%$ & Relaxed & $53 \%$ & Persuasive & $47 \%$ & Procedural & $28 \%$ \\
\hline
\end{tabular}

Source: Authors' own research results.

As it can be seen, the ideal candidate has to display honesty, flexibility, cooperativeness, efficiency, independence, confidence and coolness under pressure (see Figure 1), out of which only three (i.e., coolness under pressure) are associated with psychopathy. 


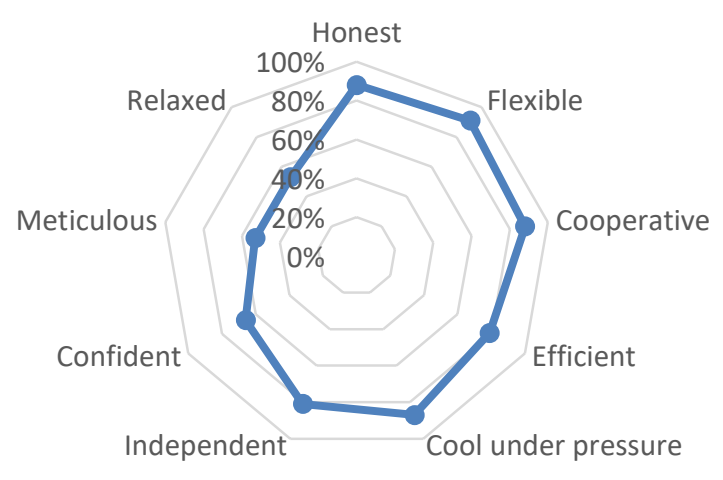

PICBE $\mid 733$

Figure 1. Desirable traits for the ideal candidate

Source: Authors' own research results.

After seeing how psychopathic traits fair against non-psychopathic traits in the preferences of interviewers, the next set of questions sought to provide a more in-depth analysis of the importance of each psychopathic trait. The responders were asked to score each trait from 1 - not important at all to 5 - highly important, based on how good of an asset that trait would be for a candidate (e.g. "During an interview, would you consider selfconfidence an important asset for a candidate?"). The results displayed in Table 3 show that the highest scoring trait was the ability to keep calm under pressure, with an average of 4.31, followed by self-confidence with an average score of 3.78 and persuasiveness with a score of 3.53. "Personal charm" was also ranked among the important traits, with an average score of 3. The three remaining traits (i.e., fearlessness, a carefree attitude, and egocentricity) received average scores below 3 , making them undesirable in job candidates.

Table 3. The importance of each psychopathic trait

\begin{tabular}{|c|c|c|c|c|c|c|c|}
\hline & $\begin{array}{c}\text { Ability to keep } \\
\text { calm under } \\
\text { pressure }\end{array}$ & $\begin{array}{c}\text { Self } \\
\text { confidence }\end{array}$ & Persuasiveness & $\begin{array}{c}\text { Personal } \\
\text { charm }\end{array}$ & Fearlessness & $\begin{array}{c}\text { A carefree } \\
\text { attitude }\end{array}$ & Egocentricity \\
\hline $\begin{array}{c}\text { Average } \\
\text { scores }\end{array}$ & $\mathbf{4 . 3 1}$ & $\mathbf{3 . 7 8}$ & $\mathbf{3 . 5 3}$ & 3 & 2.59 & 2.28 & 1.74 \\
\hline
\end{tabular}

Source: Authors' own research results.

In order to have a clear and definitive answer regarding the psychopathic traits valued by interviewers, we asked participants to choose the top 3 most important assets that a job candidate should have out of the 7 psychopathic traits. The answers confirmed the preferences previously stated, as the ability to keep calm under pressure received $94 \%$ of the votes, self-confidence $91 \%$ of the votes and persuasiveness, $63 \%$ of the votes (see Figure 2 ). Personal charm, fearlessness and carefree attitude ranked middle ground, with 32\%, 10\% and $9 \%$ of the votes respectively. Egocentricity gathered a total of 0 votes at this question, ending on the last place.

In Figure 2, we compared the consistency of the answers provided by the interviewers to the two types of questions referring to the desirability of psychopathic traits in candidates 
(the independent questions and the ranking question) and the results show that ability to keep calm under pressure and self-confidence are clearly traits valued by interviewers, followed at some distance by persuasiveness.

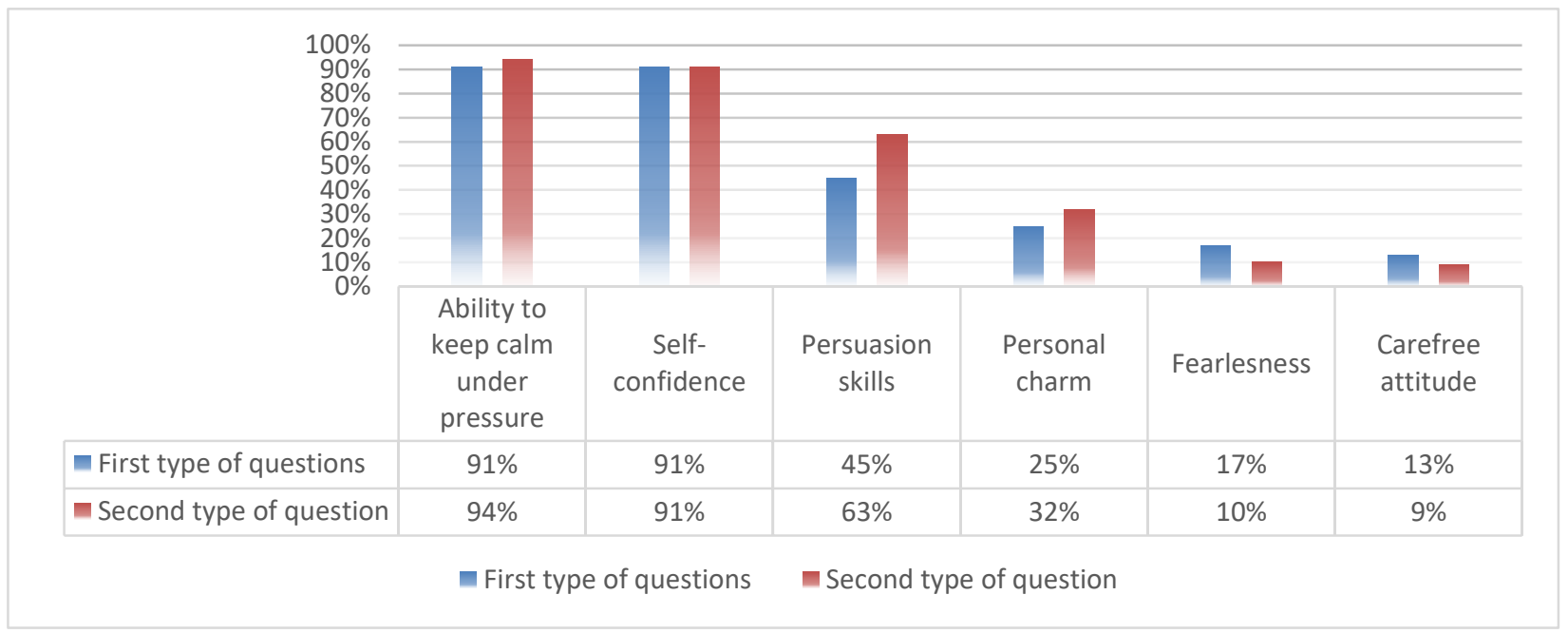

PICBE $\mid 734$

Figure 2. The consistency of the traits chosen by interviewers

Source: Authors' own research results.

In addition, we are also aware that there are certain personality characteristics which make the candidate look undesirable to interviewers. According to our literature review, the traits of psychopathic individuals that can have a negative influence on the impression made by a candidate during the selection process are: a lack of realistic long-term plans, a lack of ethics/morals, and the inability to accept responsibility for one's actions. When asked if they would reject a candidate who displayed these characteristics, the respondents stated that they would most likely reject an individual who shows a lack of ethics/morals (an average score of 4.43 out of 5) and a individual who demonstrates an inability to accept responsibility for his actions (an average score of 4.46 out of 5). In contrast, interviewers were less likely to reject candidates who display a lack of realistic long-term plans (an average score of only 2.63 out of 5 ).

As a conclusion for the first hypothesis, it has been shown that there are several psychopathic traits which are advantageous for candidates during the job interview (i.e., coolness under pressure, self-confidence and persuasiveness). In addition, we have also found that two psychopathic traits (a lack of ethics/morals and the inability to accept responsibility for one's actions) are also disadvantageous for the candidate and can lead to a rejection.

The influence of the company's field of activity on the preference for psychopathic traits This hypothesis was tested by asking participants whether their choices of the top three psychopathic traits were influenced by the daily activities performed by the company. The results in Table 4 show a comparison between participants from creative industries and those from professional services. 
Table 4. The split of answers by domain

\begin{tabular}{|c|c|c|c|c|}
\hline \multirow{2}{*}{$\begin{array}{c}\text { Did domain play an } \\
\text { important role in } \\
\text { choosing the traits? }\end{array}$} & \multicolumn{2}{|c|}{ Creative Industry } & \multicolumn{2}{c|}{ Professional Services } \\
\cline { 2 - 5 } YES & No. of people & \% of total & No. of people & \% of total \\
\hline NO & 13 & $\mathbf{8 1 . 2 5 \%}$ & 39 & $\mathbf{7 5 \%}$ \\
\hline & 3 & $18.75 \%$ & 13 & $25 \%$ \\
\hline
\end{tabular}

PICBE | 735

As can be observed, despite the difference in the number of participants, the opinions are rather similar: $81.25 \%$ of the people working in the creative industry and $75 \%$ of those in the professional services consider that their domain of work influenced to a certain extent the traits they have chosen to be beneficial for the recruiting process. Moreover, we did encounter differences in the top three traits preferred by the two groups. As shown in Table 5 , interviewers from creative industries value self-confidence over the ability to keep calm under pressure, whereas for the interviewers from professional services, the opposite is true.

Table 5. Top 3 psychopathic traits according to field of activity

\begin{tabular}{|c|c|c|c|c|c|c|}
\hline & \multicolumn{2}{|c|}{$\begin{array}{c}\text { Ability to keep calm } \\
\text { under pressure }\end{array}$} & \multicolumn{2}{c|}{ Self-confidence } & \multicolumn{2}{c|}{ Persuasion skills } \\
\cline { 2 - 7 } & $\begin{array}{c}\text { No. of } \\
\text { people }\end{array}$ & \% of total & $\begin{array}{c}\text { No. of } \\
\text { people }\end{array}$ & $\%$ of total & $\begin{array}{c}\text { No of } \\
\text { people }\end{array}$ & $\%$ of total \\
\hline $\begin{array}{c}\text { Creative } \\
\text { industry }\end{array}$ & 14 & $\mathbf{8 7 . 5 \%}$ & 16 & $\mathbf{1 0 0 \%}$ & 9 & $\mathbf{5 6 . 2 5 \%}$ \\
\hline $\begin{array}{c}\text { Professional } \\
\text { Services }\end{array}$ & 50 & $\mathbf{9 6 . 1 5 \%}$ & 46 & $\mathbf{8 8 . 4 6 \%}$ & 34 & $\mathbf{6 5 . 3 8 \%}$ \\
\hline
\end{tabular}

Source: Authors' own research results.

If taken in no particular order (because they were not asked to rank them, just choose any 3 traits from the total of 7), we observe that the same 3 traits were the same for both categories, even with the minor preferences for the first and second place. Thus, we can conclude that the company's field of activity has only a minor influence on the preference of recruiters for specific psychopathic traits in candidates.

\section{HR professionals' level of preparedness to filter out psychopathic candidates}

In order to understand whether Romanian HR professionals are able to handle the threat posed by psychopathic employees, we asked them a series of questions, starting with "Have you ever been worried that you might have hired a psychopath?". The reasoning behind this question was that, because of the fact that competent literature in the field of HR rarely touched this subject in foreign countries, and almost never in local areas, the possibility of hiring a psychopath is rarely put into question. As shown in Figure 3, 40\% of the participants answered that they had some worries, but the majority of them said that they were either not worried about this (34\%) or that they never thought about it (26\%). 


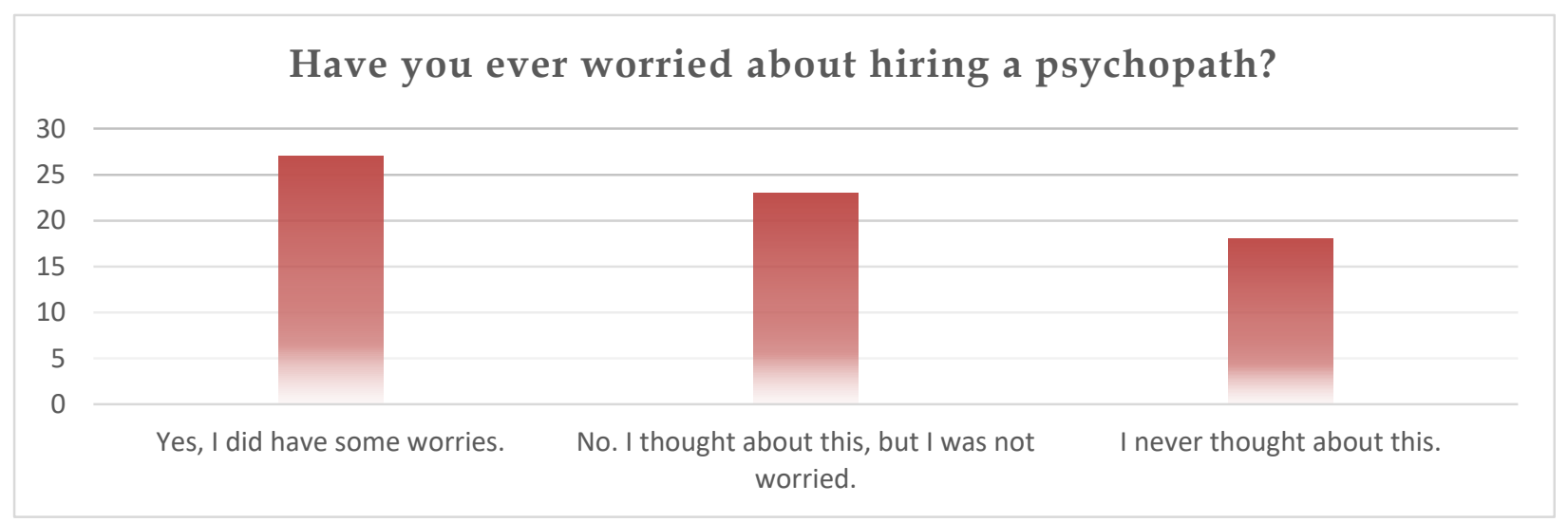

PICBE | 736

Figure 3. The awareness of HR professionals

Source: Authors' own research results.

One of the suggestions we gave in the first part of the paper was to check a candidate's background information, the previous experience and such. This is useful because psychopaths, being notorious liars, might insert into their CVs parts that are not true, or are exaggerated, just be to seem more prepared for the job ahead. The use of background checks was fairly common among the participants, as the most of them said that they use background checks for both low-level and high-level positions (44\%), while another 37\% used background checks only for high-level positions (see Figure 4). 13 participants reported that they do not use background checks.

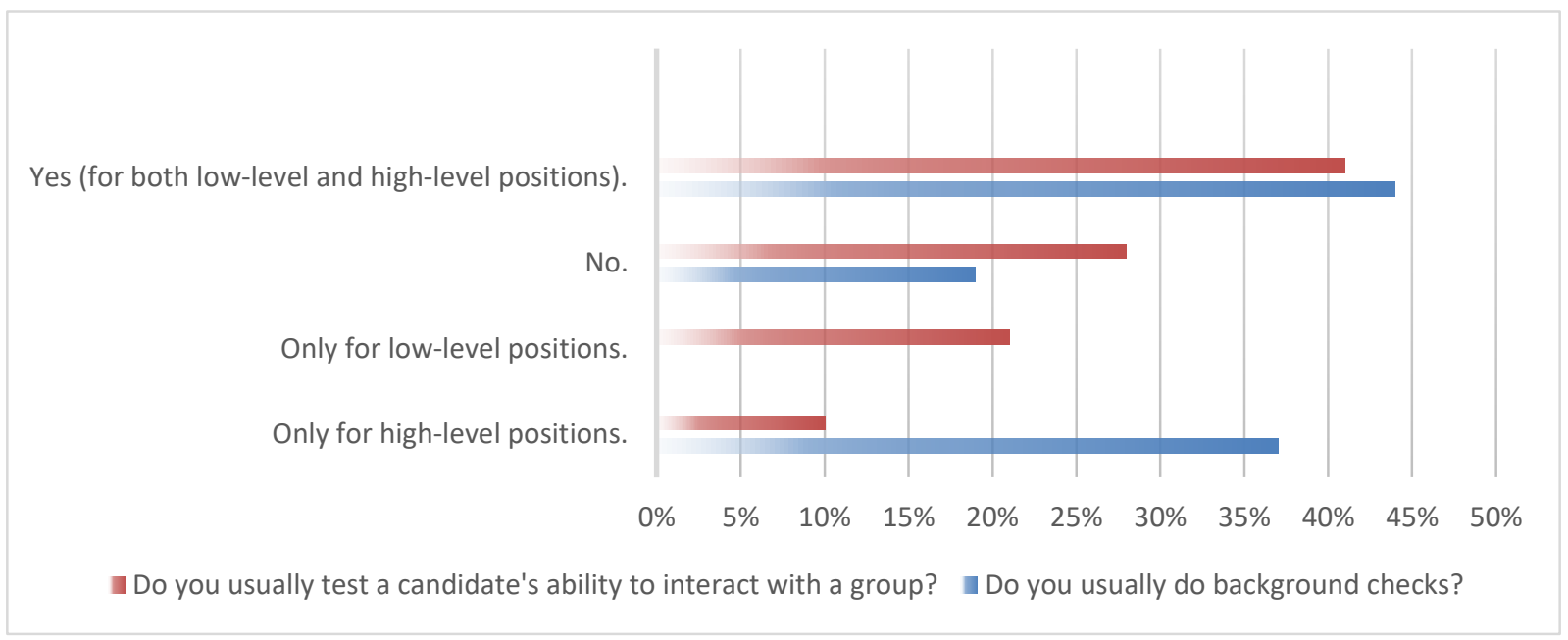

Figure 4. The check against psychopathic traits

Source: Authors' own research results.

Another suggestion for filtering out psychopaths was to test an individual's ability to interact with a group. Because psychopaths are usually capable of feeding a different type of story to different people, because their ability to "read" a person, or because they have a more aggressive behavior towards people they see as useless towards their cause, having them put

DOI: 10.2478/picbe-2019-0065, pp. 728-739, ISSN 2558-9652| Proceedings of the $13^{\text {th }}$ International Conference on Business Excellence 2019 
into a group and seeing how they interact with others might give the recruiter some hints about the personality of the individual. Thus, we asked participants if they test the candidate's ability to interact with other people, and $41 \%$ answered that everyone in their selection process has to interact with a group, while $28 \%$ admitted they do not test against that. $21 \%$ of the participants only tested candidates for low-level positions, while only $10 \%$ of the participants tested only candidates for high-level positions. In conclusion, the selection process in the majority of cases contained at least two tests that could reveal the presence of psychopathic characteristics.

\section{Conclusion}

The aim of the study was to establish which psychopathic traits are considered desirable by interviewers and if the selection processes used by Romanian HR specialists from two specific fields are effective in filtering out candidates with psychopathic features. Psychopathy does not have a clear, all-inclusive and universally accepted definition, nor is it just a single trait which generates a clear difference between individuals. Instead, it is rather a constellation of traits from which each person can have from as little as one trait to the full spectrum of characteristics. Depending on each individual's personal background and severity of the inclination towards psychopathy, these traits could help him get ahead of others or get him behind bars.

Successful psychopaths, the so-called functional psychopaths, are people who do not exhibit the whole spectrum of traits, or who have learned how to limit their negative impulses - a surgeon who uses his cold-blood to save people, a former special forces agent who channeled his psychopathic traits into doing good, or businessmen affected by pathological lying and lack of remorse who climbed the corporate ladder and just caused the world economic crisis with the smile on their face. Thus, it is important to understand the effects these individuals have over the business environment, how their peculiar personality and way of acting can damage a company's culture if they succeed to reach managerial positions, or how they can make the lives of the people around them significantly harder. We focused on the way in which HR specialists can filter psychopathic candidates and explored both the methods used for selecting personnel and the desirability of psychopathic traits in candidates.

We asked HR professionals their opinion regarding the existence of certain psychopathic traits useful when it comes to landing a job and we found that there are three psychopathic traits which advantage candidates: ability to remain calm under pressure, selfconfidence and persuasiveness. This insight is useful both for future candidates who can nurture these skills in order to increase their employability and for HR professionals who can analyze their own biases and learn how to discern danger signals from candidates that display a certain array of positively valued psychological traits but which can hide a psychopathic tendency. We have also learned that the employer 's field of activity play little to no role in the preference that HR professionals have towards the psychopathic traits displayed by candidates. Finally, we found that Romanian HR professionals are somewhat aware of the threat posed by psychopathic employees and that most of the employers they represent use selection methods able to filter out potential psychopaths.

This is the first study of the perceptions of HR professionals of the desirability of psychopathic traits in job candidates from Romania, and, although exploratory in nature, it is 
significant because it raises awareness of the importance of this topic and serves as a stepping stone for future research.

\section{References}

Babiak, P., \& Hare, R. (2006). Snakes in suits: When psychopaths go to work. New York: HarperCollins.

Babiak, P., Neumann, C. \& Hare, R. (2010). Corporate psychopathy: Talking the walk. Behavioral Sciences \& the Law, 28(2), 174-193.

Ballantine, I. (2009). Recruiting and selecting staff in organizations. In: Gilmore, S. (Ed.) Human resource management (pp. 92-116). Oxford: Oxford University Press.

Board, B.J., \& Fritzon, K. (2005). Disordered personalities at work. Psychology, Crime \& Law, 11(1), 17-32.

Boddy, C.R. (2011). Corporate psychopaths: Organisational destroyers. London: Palgrave MacMillan.

Boddy, C.R. (2011). The corporate psychopaths theory of the global financial crisis. Journal of Business Ethics, 102(2), 255-259.

Boddy, C.R.P., Ladyshewsky, R., \& Galvin, P. (2010a). Leaders without ethics in global business: Corporate psychopaths. Journal of Public Affairs, 10(3), 121-138.

Boddy, C.R., Ladyshewsky, R.K., \& Galvin, P. (2010b). The influence of corporate psychopaths on corporate social responsibility and organizational commitment to employees. Journal of Business Ethics, 97(1), 1-19.

Bratton, J., \& Gold, J. (2012). Human resource management: theory and practice. London: Palgrave Macmillan.

Cleckley, H. (1941). The mask of sanity. $3^{\text {rd }}$ ed. St Louis: C.V. Mosby.

Deresiewicz, W. (2012, May 13). Capitalists and other psychopaths. Retrieved from: www.nytimes.com/2012/05/13/opinion/sunday/fables-of-wealth.html.

Dutton, K. (2012). The wisdom of psychopaths: What saints, spies, and serial killers can teach us about success. New York: Farrar, Straus and Giroux.

Faggioni, M., \& White, M. (2009). Organizational psychopaths - Who are they and how to protect your organization from them. Sudbury: Integrity Management Consulting Group.

Hare, R. (1980). A research scale for the assessment of psychopathy in criminal populations. Personality and Individual Differences, 1(2), 111-119.

Hare, R. (2003). The Hare psychopathy checklist-Revised. $2^{\text {nd }}$ ed. Toronto: Multi-Health Systems.

Koch, J.L.A. (1892). Die psychopathischen minderwertigkeiten. Retrieved from: archive.org/details/diepsychopathisc00koch/page/n8.

Lilienfeld, S.O., \& Andrews, B. (1996). Development and preliminary validation of a selfreport measure of psychopathic personality traits in noncriminal populations. Journal of Personality Assessment, 66(3), 488-524.

Lilienfeld, S.O., Waldman, I.D., Landfield, K., Watts, A.L., Rubenzer, S., Faschingbauer, T.R. (2010). Fearless dominance and the U.S. presidency: Implications of psychopathic personality traits for successful and unsuccessful political leadership. Journal of Personality and Social Psychology, 103(3), 489-505. 
Lilienfeld, S.O., \& Widows, M.R. (2005). Psychopathic Personality Inventory—Revised (PPI-R) professional manual. Lutz, FL.

Millon, T., Simonsen, E., Birket-Smith, M., \& Davis, R.D. (1998). Psychopathy: Antisocial, criminal, and violent behavior. New York: The Guilford Press.

Patrick, C.J., Fowles, D.C., \& Krueger, R.F. (2009). Triarchic conceptualization of psychopathy: Developmental origins of disinhibition, boldness, and meanness.

PICBE | 739

Pritchard, J. C., 1835. A treatise on insanity and other disorders affecting the mind. Development and Psychopathology, 21(3), 913-938.

Skeem, J., Polaschek, D., Patrick, C., \& Lilienfeld, S. (2011). Psychopathic personality: Bridging the gap between scientific evidence and public policy. Psychological Science in the Public Interest, 12(3), 95-162.

Smith, S.F., \& Lilienfeld, S.O. (2012). Psychopathy in the workplace: The knowns and unknowns. Aggression and Violent Behavior, 18(2), 204-218.

Smith, S.F., Lilienfeld, S.O., Coffey, K., \& Dabbs, J.M. (2013). Are psychopaths and heroes twigs off the same branch? Evidence from college, community, and presidential samples. Journal of Research in Personality, 47(5), 634-646.

Verhaeghe, P. (2014, September 29). Neoliberalism has brought out the worst in us. Retrieved from: www.theguardian.com/commentisfree/2014/sep/29/neoliberalismeconomic-system-ethics-personality-psychopathicsthic. 